Canadian Oncology

Nursing Journal

Revue canadienne

de soins infirmiers

en oncologie

Volume 30, Issue 2 • Spring 2020

elSSN: 2368-8076 


\title{
Besoins des proches aidants qui accompagnent une personne en soins palliatifs et de fin de vie à domicile
}

\author{
par Elizabeth Pepin et Johanne Hébert
}

\section{RÉSUMÉ}

Le maintien à domicile d'une personne en soins palliatifs et de fin de vie (SPFV) dépend grandement de la présence quotidienne de proches aidants (PA) et de leur implication dans les soins. Or, les besoins des PA tout au long de la trajectoire d'accompagnement d'un proche en SPFV à domicile sont encore relativement méconnus.

Objectifs et méthode : Cette étude qualitative descriptive s'intéresse au rôle des PA qui ont accompagné une personne recevant des SPFV à domicile dans le but de décrire leurs besoins tout au long de la trajectoire d'accompagnement. Ainsi, 20 PA ont participé à des entrevues semi-dirigées.

Résultats et discussion : Létude met en lumière les besoins multiples des PA qui accompagnent un proche en SPFV à domicile. Les besoins informationnels, émotionnels et psychosociaux démontrent que les PA connaissent des changements dans leur rôle auprès de la personne malade. Les besoins spirituels ont été rapportés en termes de sens donné à l'expérience d'accompagnement. Enfin, les besoins pratiques mettent de l'avant l'importance d'avoir accès aux services de SPFV $24 \mathrm{~h} / 24$ et le caractère essentiel du soutien de linfirmière.

Conclusion: Les besoins des PA qui accompagnent une personne en SPFV à domicile sont peu satisfaits. Il importe d'en tenir compte dans la trajectoire de soins, parallèlement aux besoins de la personne malade, afin d'améliorer l'expérience d'accompagnement qui précède la période de deuil.

Mots clés : transition, besoins, proches aidants, soins palliatifs et de fin de vie, domicile, rôle infirmier

\section{INTRODUCTION}

L'Organisation mondiale de la santé (OMS/WHO, 2018) définit les soins palliatifs (SP) comme les soins qui améliorent la qualité de vie d'une personne et de ses proches confrontés aux problèmes d'ordre physique, psychosocial et spirituel

\section{AUTEURES}

Elizabeth Pepin, infirmière clinicienne, étudiante à la maîtrise, Université du Québec à Rimouski, campus de Lévis, Maison Michel-Sarrazin

Elizabeth.Pepin@uqar.ca

Johanne Hébert, infirmière, Ph.D., Directrice de recherche, Université du Québec à Rimouski, campus de Lévis, Maison Michel-Sarrazin, Centre intégré de santé et de services sociaux de Chaudière-Appalaches

Johanne_Hebert@uqar.ca associés à une maladie incurable, sans hâter ni retarder la mort. Ces soins préviennent et soulagent la souffrance grâce à l'évaluation et au traitement précoces de la douleur et des symptômes. Au Québec, la Loi concernant les soins de fin de vie assure aux personnes en fin de vie l'accès à des soins de qualité qui répondent à leurs besoins tout au long de la trajectoire de soins, dans le respect de l'autonomie et de la dignité et en vue d'aider à prévenir et à soulager la souffrance (Légis Québec, 2018). La majorité des personnes en fin de vie mentionnent qu'elles aimeraient décéder dans le confort de leur domicile (SCC, 2013) si leur situation le permet. Toutefois, près de $80 \%$ des décès surviennent en milieu hospitalier (Statistique Canada, 2019b). Dans son plan de développement 2015-2020 concernant les SPFV, le ministère de la Santé et des Services sociaux du Québec (2015) priorise le maintien à domicile si tel est le souhait de la personne et de ses proches.

De fait, le maintien à domicile dépend grandement de l'implication des proches aidants (PA), ceux qui offrent du soutien à un proche ayant une incapacité significative et persistante susceptible de compromettre le maintien à domicile (L'Appui pour les proches aidants d'aînés, 2012). De nombreuses personnes rapportent qu'elles auraient aimé offrir les soins palliatifs et de fin de vie (SPFV) à domicile, mais que des contraintes - notamment le manque de soutien et de ressources et le fait de devoir s'absenter du travail - les en ont empêchés (L'Appui pour les proches aidants d'aînés, 2012). La recension des écrits a permis de constater que pour se préparer à leur rôle, les PA ont besoin d'informations concernant la maladie de leur proche, particulièrement en ce qui concerne la gestion des symptômes, les soins à offrir (Funk et al., 2015) et la gestion et l'administration de la médication (Wilson et al., 2018). Pour ce faire, ils ont besoin d'être guidés et informés par les professionnels de la santé (notamment l'infirmière) afin de diminuer l'anxiété liée à l'exécution de ces tâches (Sheehy-Skeffington et al., 2014). Plusieurs PA ont fait l'expérience d'être présents $24 \mathrm{~h} / 24$ afin d'assumer les responsabilités de soins en plus d'effectuer les tâches de la vie quotidienne (Robinson et al., 2017). Cet accompagnement génère un profond sentiment d'isolement et est source d'anxiété (Totman et al., 2015). De plus, les PA rapportent la difficulté de s'adapter aux changements dans la relation lorsque le rôle de soignant devient prépondérant, redéfinissant ainsi la nature de la relation (Totman et al., 2015). Enfin, les PA expriment des craintes par rapport au processus de fin de vie (Soroka et al., 2018) et constatent un manque de préparation au deuil (Mason et Hodgkin, 2019). 


\section{CADRE CONCEPTUEL DE L'ÉTUDE}

Pour décrire les besoins associés à l'expérience de transition vers le rôle de PA, le cadre conceptuel s'appuie notamment sur la théorie intermédiaire de la transition (Meleis, 2010) et le cadre des soins de soutien en oncologie (Fitch et al., 2008). Ainsi adapté aux concepts de l'étude, il permet de mieux comprendre les éléments qui facilitent ou entravent la transition vers le rôle de PA et d'identifier les besoins qui en découlent (Figure 1).

Dans cette étude, la transition se caractérise par le passage d'un rôle à un autre. La transition vers le rôle de PA indique un changement dans les relations, dans les attentes liées à ce rôle ou encore dans la capacité à accomplir les responsabilités liées au rôle (Meleis, 2010). Cette transition de nature situationnelle débute généralement lors de l'annonce d'une maladie grave et de l'apparition de symptômes chez le proche malade, et perturbe le sentiment de normalité et d'équilibre des PA (Penrod et al., 2012). De fait, prendre soin d'une personne en SPFV à domicile engendre de nombreux défis auxquels les PA doivent s'adapter tant sur les plans physique, informationnel, émotionnel ou psychologique que social et spirituel (Carlander et al., 2011).

\section{BUT ET OBJECTIFS DE L'ÉTUDE}

Cette étude qualitative descriptive s'intéresse au rôle des proches aidants (PA) qui ont accompagné une personne recevant des soins palliatifs et de fin de vie (SPFV) à domicile. L'objectif poursuivi est de décrire les besoins des PA qui accompagnent une personne en SPFV à domicile.

\section{MÉTHODOLOGIE}

Cette étude s'insère dans un projet de recherche plus large s'intitulant «Conditions favorisant et limitant le maintien à domicile en SPFV » (Hébert et al., 2017). Elle s'intéresse à décrire les besoins des personnes qui ont fait l'expérience de transition vers le rôle de PA en accompagnant une personne en SPFV à domicile. L'approche qualitative descriptive a été privilégiée. Afin d'assurer la transférabilité des données, l'échantillon était constitué de PA provenant de deux secteurs différents afin de représenter les milieux rural et urbain. La crédibilité et la fiabilité de l'étude ont été assurées par la triangulation de la recension des écrits scientifiques et de la littérature grise concernant les SPFV à domicile avec les besoins des PA rapportés dans l'étude. De plus, les résultats ont pu être comparés avec ceux de l'étude principale afin de vérifier la validité des résultats. Enfin, un exercice de validation des résultats a permis d'assurer la confirmabilité des résultats. Dans un premier temps, l'étudiante a encodé l'ensemble des données des entrevues. Par la suite, un tableau rassemblant tous les verbatims encodés et un tableau indépendant comprenant l'ensemble des codes utilisés ont été créés. Une professionnelle de recherche et la directrice de recherche ont fait l'exercice d'encoder les citations des verbatims pour valider les codes afin d'assurer l'objectivité de l'interprétation des données.

\section{Participants et procédure}

Un échantillon de type intentionnel a été constitué. Pour être admissible, les PA devaient répondre aux critères suivants : 1) être âgés de 18 ans et plus; et 2) avoir accompagné une personne qui avait reçu des services de SPFV à domicile dans les deux dernières années. Des entrevues semi-dirigées ont été réalisées à l'aide d'un guide d'entrevue élaboré en prenant en compte le cadre conceptuel de l'étude principale (Fillion, Veillette, Wilson, Dumont et Lavoie, 2009; Gomes et Higginson, 2006; Stewart, Teno, Patrick et Lynn, 1999) et portant sur les thèmes suivants : 1) les connaissances et perceptions associées aux SPFV; 2) les besoins des personnes en fin

Intégration des concepts de Meleis et Fitch

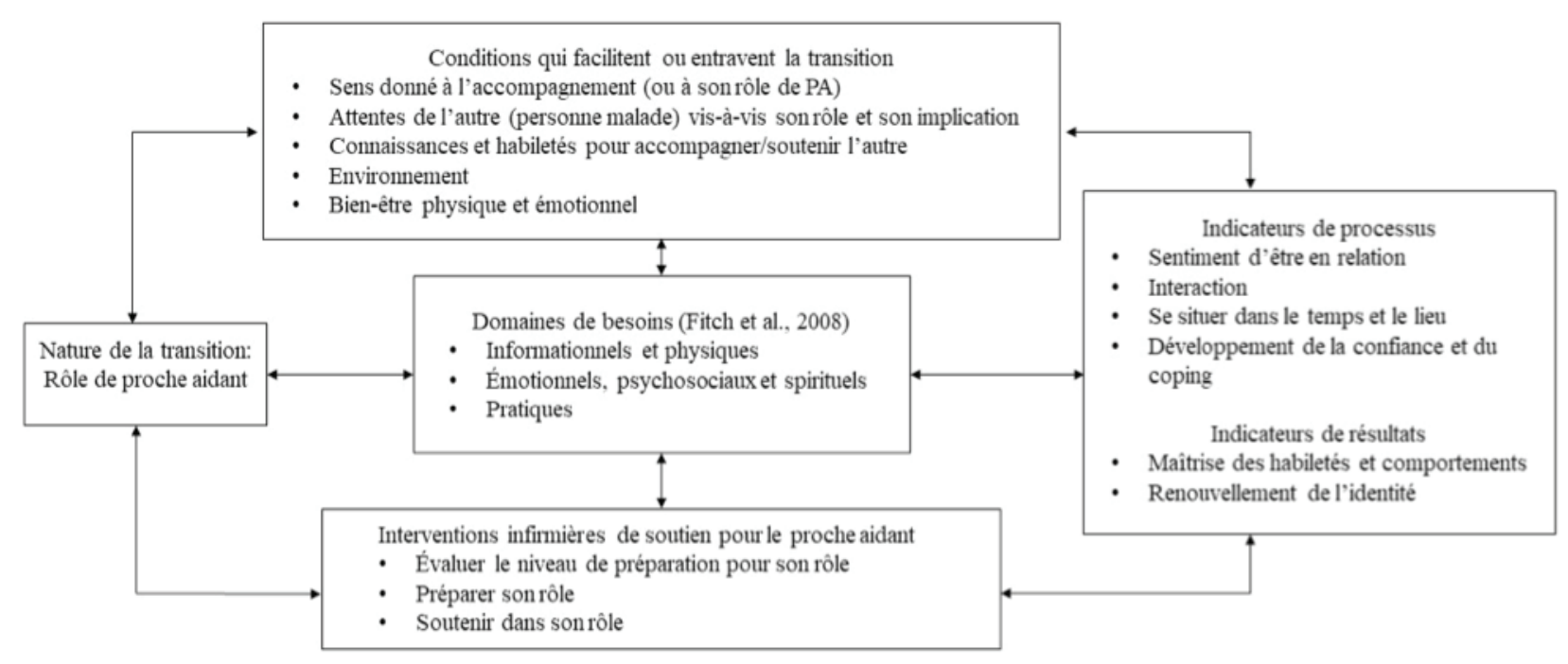

Figure 1. Intégration des modèles de Meleis (2010) et de Fitch et al. (2008). 
de vie à domicile et de leurs proches; et 3) les principaux défis à relever pour l'amélioration des SPFV à domicile. Chacun de ces thèmes regroupait des sous-questions qui permettaient d'approfondir l'expérience de SPFV à domicile vécue. Les entretiens ont été audio-enregistrés avec l'accord écrit des participants. Les entretiens se sont déroulés au domicile des participants afin qu'ils se sentent à l'aise de partager leur expérience. Cette étude a été approuvée par le comité éthique de la recherche d'un centre intégré de santé et de services sociaux.

\section{Analyse des données}

Dans le but de décrire les besoins des PA dans la transition vers leur rôle d'accompagnement d'un proche en SPFV à domicile, les données ont été organisées en fonction des domaines de besoins présentés dans le cadre des soins de soutien en oncologie (Fitch et al., 2008). L'analyse des données d'entrevues a été réalisée selon l'approche de Miles et Huberman (2003), qui préconise une démarche en trois étapes : 1) la condensation des données (organisation des données); 2) la présentation (assemblage organisé de l'information tirée des données); et 3) l'élaboration/vérification des données (conclusions finales de l'ensemble des données). À la suite de la transcription intégrale des entrevues en verbatim et de leur lecture approfondie, les thèmes émergents ont été organisés en unité de sens et codifiés à l'aide du logiciel NVivo 10.

\section{RÉSULTATS}

\section{Besoins de soutien des proches aidants}

Concernant les besoins informationnels, plusieurs PA ont mentionné qu'ils avaient peur de ne pas avoir les connaissances nécessaires pour prendre soin de leur proche à domicile. Ainsi, le soutien des professionnels de la santé a été souligné comme étant essentiel. De fait, les PA ont indiqué avoir besoin d'informations pour la gestion de la douleur concernant, par exemple, les procédures à suivre pour les injections de médicaments en vue d'assurer un soulagement optimal de la douleur: "Nous faisions les injections de médicaments, même ma petite-fille en a fait. L'infirmière nous a vraiment bien expliqué, c'était facile » (PA 15). Une mauvaise gestion des symptômes ou un manque de connaissances de ceux-ci génèrent de l'anxiété et amènent les $\mathrm{PA}$ à recourir aux services d'urgence. Il semble primordial d'avoir une personne-ressource vers qui se tourner afin d'obtenir l'information et le soutien nécessaires : « [...] quand il $y$ a des crises la nuit, de la douleur, les médicaments qui donnaient des effets secondaires [...] on se pose des questions quand on ne connaît pas ça [...] c'est important d'avoir un soutien, d'avoir une personne à qui nous pouvons poser des questions » (PA 15). Les infirmières et les travailleurs sociaux étaient les professionnels les plus souvent mentionnés par les PA.

Les besoins physiques ont révélé que l'accompagnement du proche malade dans les soins quotidiens à offrir (gestion de la médication, soins d'hygiène, préparation des repas) 24 heures par jour engendre un manque de sommeil. Des participants ont souligné que l'épuisement et l'augmentation constante des responsabilités les ont amenés à devoir transférer leur proche malade dans un autre établissement : «À la fin, nous aurions eu besoin d'avoir quelqu'un à temps plein pour nous aider, car nous étions tous exténués. On allait chez (personne décédée), on ne dormait pas parce qu'il ne dormait pas, nous étions inquiets et nous avons continué à travailler le jour [...]. Nous avons fait trois semaines comme ça et ensuite nous avons accepté un lit en maison de soins palliatifs » (PA 18). De fait, le besoin d'avoir du soutien pendant la nuit pour se reposer et être en mesure d'offrir de meilleurs soins le jour était un élément fréquemment rapporté. De plus, les PA ont constaté les conséquences de leur expérience d'accompagnement pendant plusieurs mois après le décès de leur proche.

Par la suite, plusieurs besoins émotionnels, psychosociaux et spirituels ont été rapportés. L'incertitude face à l'avenir est une source d'anxiété pour les PA. «Savoir qu'elle dégradait, de voir qu'elle en perdait [...] lorsque je savais que ça n'allait plus, les derniers six mois ont été difficiles, de ne pas savoir, c'est limproviste» (PA 14).

Ainsi, plus la maladie évolue, plus ils ont de la difficulté à quitter leur proche pour prendre du répit. Bien que le besoin de répit soit très présent, ils expriment paradoxalement le besoin de passer le plus de temps possible avec leur proche, car ce temps est limité : «[...] c'est bizarre parce que parfois, on se dit qu'avoir quelqu'un pour la nuit pour donner un peu de répit aurait fait du bien, mais en même temps nous sommes tellement impliqués qu'on ne veut rien manquer, qu'on sent que c'est nous les seuls qui pouvons le faire [...] il me semble que je suis la mieux placée » (PA 11). De plus, la relation de confiance et de proximité qui s'établit fait en sorte qu'il devient difficile pour le PA de confier les soins à d'autres personnes.

Par ailleurs, être PA transforme la relation de conjoint(e) ou d'enfant à celle de soignant et a amène les PA à mettre leur vie de côté pour prendre soin de leur proche : « Quand ça fait 35 ans que tu vis avec la même personne et que tu ne la reconnais plus, c'est difficile. J'étais l'amoureux qui devenait l'infirmier, le préposé, l'homme à tout faire, j'étais l'homme de ménage " (PA 17). Cependant, le partage des responsabilités avec d'autres membres de la famille et la présence d'amis ont été soulevés comme étant bénéfiques pour diminuer le sentiment de fardeau et d'isolement.

Les PA ayant accompagné un proche atteint d'une autre maladie que le cancer rapportent qu'ils avaient eu peu d'accès à des ressources, un fait souvent dû au manque de connaissances des professionnels de la santé concernant la maladie de leur proche : «On a fait une demande à un endroit, mais ils n'avaient pas l'air d'accepter les personnes qui n'avaient pas le cancer [...] parce que lorsque j'ai parlé de la maladie, oups j'ai vu que c'était un peu le néant pour eux » (PA 14).

Finalement, les besoins spirituels se sont exprimés par la signification donnée à l'expérience d'accompagnement. La plupart des PA ont rapporté avoir réfléchi aux priorités et réalisé l'importance de vivre le moment présent et d'apprécier les choses simples de la vie. Plusieurs ont perçu l'expérience d'accompagnement comme un privilège : « [...] c'était un privilège d'être proche de mon père pour réaliser son souhait de mourir à la maison, c'est le plus beau cadeau qu'on ait pu lui faire qu'il puisse mourir en toute dignité, c'était vraiment quelque chose que je voulais lui donner » (PA 11). Ils soutiennent également que le 
fait d'être restés à domicile jusqu'au décès a conféré un caractère plus humain à leur expérience, ce que l'effervescence des hôpitaux n'aurait pas pu leur offrir. D'autres ont mentionné qu'ils avaient eu peur que le décès à domicile laisse des images difficiles à effacer et ont choisi un autre lieu pour le décès. La présence de l'infirmière après le décès a contribué à une expérience positive pour les PA : «Ce que j'ai trouvé le plus humain, c'est que suite au décès, nous décidions du moment où nous appelions l'infirmière de garde. Ensuite, l'infirmière vient [...] il y a eu un grand respect de la personne, c'était tellement beau, elle a pris le temps et ensuite elle a fait la liaison avec le médecin pour le constat de décès » (PA 11).

Enfin, les PA ont rapporté un sentiment de vide à la suite du décès de leur proche : «Ça été très long sortir de cette vie-là. Nous vivions une vie entre parenthèse [...] et quand on sort de tout ça après le décès, c'est très long avant de revenir à une vie normale » (PA 18). Ils soulignent qu'ils auraient apprécié recevoir du soutien de la part des professionnels de la santé après le décès de leur proche.

Les besoins pratiques ont révélé que la stabilité de l'équipe de soins permettait aux PA de développer une relation de confiance avec les professionnels de la santé et favorisait un meilleur suivi de l'évolution de l'état de santé de leur proche : « L'infirmière venait ici régulièrement, il était en confiance avec elle [...] elle faisait des suggestions durant la progression de la maladie parce qu'elle savait qu'il n'accepterait pas tout de suite le soutien proposé [...] et le moment venu, il disait oui tout de suite » (PA 10). L'accès aux services 24 heures/24 et 7 jours par semaine a également été rapporté comme essentiel à la qualité du suivi. Inversement, la difficulté d'accès aux services en temps opportun laissait aux PA le sentiment d'être laissés à eux-mêmes et isolés : "À la fin, j'avais besoin de plus de soutien mais je n'ai pas pu en avoir [...] il n'y avait personne pour venir parce que je vis en région [...] on se sent seul à ce moment-là » (PA 7). À cet effet, la présence de l'infirmière était d'une aide précieuse pour les soutenir dans leurs responsabilités de soins au quotidien : "Il y a eu une période où les infirmières venaient deux fois par jour pour le changement des pansements ou simplement pour s'assurer que tout se passait bien... d'autres fois, elles nous appelaient. C'était rassurant » (PA 19). De plus, la présence du travailleur social permettait de faire le lien entre les différents intervenants et d'intégrer de nouveaux services facilitant le maintien à domicile.

\section{DISCUSSION}

Cette étude avait pour but de mieux comprendre les besoins des PA qui accompagnent un proche en SPFV et de décrire le rôle de soutien de l'infirmière dans ce contexte. La théorie intermédiaire de la transition (Meleis, 2010) a permis de porter une attention particulière aux conditions qui facilitent et entravent la transition vers le rôle de PA tout au long de la trajectoire d'accompagnement. En effet, l'adaptation à ce rôle est un processus continu pendant lequel de nombreux besoins émergent.

Ainsi, le cadre des soins de soutien en oncologie (Fitch et al., 2008) a permis d'abord de préciser les besoins pour lesquels les PA avaient besoin de soutien et d'explorer les interventions pour tenter d'y répondre. Les besoins informationnels précisent l'importance de l'accès à l'information afin de pouvoir gérer les différents aspects de la maladie du proche malade, notamment en ce qui concerne les symptômes de la maladie et la médication. À cet effet, le soutien de l'infirmière est essentiel. Funk et al. (2015) rapportent que l'accès à l'information pertinente en temps opportun aide à comprendre et à accepter le diagnostic de la maladie terminale. De plus, la prise en charge des soins a un impact sur les besoins physiques des PA. En effet, le fait d'assurer une présence constante réduit le nombre d'heures de sommeil et contribue à augmenter la fatigue. Harding et al. (2012) constatent également que certains problèmes de santé comme les maladies cardiaques limitaient la capacité des PA à prendre en charge les soins de leur proche. Toutefois, ce sujet a peu été abordé par les PA de cette étude.

Les besoins émotionnels et psychosociaux ont permis de préciser les changements qui surviennent dans la relation entre les PA et la personne malade, notamment dans la transition vers le rôle de soignant. Les PA mentionnent de façon récurrente avoir mis de côté leur propre vie afin de pouvoir prendre soin de leur proche. De fait, Horseman et al. (2019) précisent que les PA font abstraction de leurs besoins personnels, ce qui nuit à la reconnaissance de leurs besoins de soutien. Par ailleurs, les PA rapportent que la relation qui les unit à leur proche se transforme, laissant de côté le rôle de conjoint(e) ou d'enfant pour prendre celui de soignant. Holm et collaborateurs (2015) précisent que l'adaptation à ce nouveau rôle est un processus continu qui se fait tout au long de la trajectoire d'accompagnement.

Enfin, les besoins spirituels ont démontré que de prendre soin d'un proche en SPFV permet aux PA de revoir leurs priorités de vie. L'expérience d'accompagnement est décrite par plusieurs comme un privilège et le domicile confère un caractère plus humain à leur expérience, en offrant davantage d'intimité. Tout comme dans l'étude de Hasson et al. (2010), les PA rapportent un profond sentiment de vide à la suite du décès de leur proche et mentionnent avoir besoin du soutien des professionnels de la santé durant la période du deuil. Toutefois, ce soutien semble absent. Johnson (2015) mentionne qu'il relève du rôle de l'infirmière d'accompagner les PA lors de la période de deuil, en collaboration avec l'équipe interprofessionnelle.

Les besoins pratiques révèlent que pour les PA, il est essentiel de recevoir du soutien de la part des professionnels de la santé et ce, 24 h/24 et 7 jours/7. La continuité des soins permet aux PA de développer une relation de confiance avec les infirmières et favorise un meilleur suivi des changements dans l'état de santé du proche malade. Bien que certaines études rapportent un changement fréquent des intervenants (Seamark et al., 2014), les PA de cette étude ont rapporté peu de changement dans les intervenants, ce qui était rassurant. La présence de l'infirmière permet aux PA de développer leurs connaissances au sujet des soins et de valider leurs interventions auprès de leur proche. Wahid et collaborateurs (2018) rapportent que le manque de soutien des professionnels de la santé diminue la confiance en soi des PA et favorise le recours aux services d'urgences. Les PA mentionnent également la 
nécessité d'avoir du soutien pour effectuer les tâches domestiques telles que le ménage et la préparation des repas, car la prise en charge des soins de leur proche est omniprésente. En effet, Ewing et Grande (2012) ont constaté que l'exécution de ces tâches ajoute à l'anxiété des PA, particulièrement lorsqu'ils ont un emploi. Le partage des responsabilités avec les membres de la famille et les amis est donc essentiel et permet de diminuer le sentiment d'isolement (Totman et al., 2015). Bien que le besoin d'avoir des moments de répit est primordial pour être en mesure de prendre soin du proche malade, les PA expriment également vouloir passer le plus de temps possible avec leur proche.

Dans cette étude, l'inégalité dans l'accès aux soins a été rapportée concernant l'accompagnement en SPFV à domicile d'un proche atteint d'une autre maladie que le cancer. Le refus d'accès à certains services (comme l'admission en maison de soins palliatifs) et le manque de connaissances des professionnels de la santé concernant la maladie et les services disponibles ont été mentionnés. D’autres études soutiennent que cette inégalité est due en partie à la perception que les SPFV sont destinés aux personnes atteintes de cancer (Hasson et al., 2010) et au manque de connaissances des professionnels de la santé (Aoun et al., 2017) qui engendrent une référence tardive vers les services appropriés. Ainsi, une approche palliative intégrée précoce de soins palliatifs permettrait une meilleure réponse aux besoins des personnes et de leur famille tout au long de la trajectoire de la maladie et contribuerait à améliorer la qualité de vie (ACSP et La Coalition pour des soins de fin de vie de qualité au Canada, 2015).

\section{RÉFÉRENCES}

Aoun, S., Deas, K., Kristjanson, L. J., \& Kissane, D. W. (2017). Identifying and addressing the support needs of family caregivers of people with motor neurone disease using the Carer Support Needs Assessment Tool. Palliative Q Supportive Care, 15(1), 32-43. https://doi.org/10.1017/S1478951516000341

ASCP/CHPCA. Association canadienne de soins palliatifs/Canadian Hospice Palliative Care Association \& La Coalition pour des soins de fin de vie de qualité au Canada. (2015). Cadre national «Aller de l'avant »: feuille de route pour l'intégration de l'approche palliative, Initiative Aller de l'avant: des soins qui intègrent l'approche palliative. Gouvernement du Canada

Carlander, I., Sahlberg-Blom, E., Hellström, I., \& Ternestedt, B.-M. (2011). The modified self: Family caregivers' experiences of caring for a dying family member at home. Journal of Clinical Nursing, 20(7-8), 1097-1105. https://doi. org/10.1111/j.1365-2702.2010.03331.x

Ewing, G., \& Grande, G. (2012). Development of a Carer Support Needs Assessment Tool (CSNAT) for end-of-life care practice at home: A qualitative study. Palliative Medicine, 27(3), 244-256. https://doi.org/10.1177/0269216312440607

Fillion, L., Veillette, A., Wilson, D., Dumont, S., \& Lavoie, S. (2009). Les racines de la «Belle Mort » : Une étude ethnographique en milieu rural au Québec. Les Cahiers francophones de soins palliatifs, 9, 61-74.

Fitch, M. I., Porter, H. B., \& Page, B. D. (2008). Supportive care framework: A foundation for person-centred care. Pappin Communications.

\section{LIMITES DE L'ÉTUDE}

Cette étude comporte certaines limites. D'abord, la taille de l'échantillon ne permet pas de généraliser les résultats concernant les besoins de soutien des PA qui accompagnent une personne en SPFV à domicile à l'ensemble des PA. De plus, seulement un PA avait accompagné une personne atteinte d'une autre maladie que le cancer, ce qui ne permet pas de valider si les besoins sont similaires durant la trajectoire de la maladie. Par ailleurs, la majorité des PA rencontrés dans cette étude étaient endeuillés, ce qui a pu influencer leurs perceptions de l'expérience vécue.

\section{CONCLUSION}

Les résultats de cette étude mettent en lumière les multiples besoins des PA et la nécessité d'y répondre pour favoriser le maintien et le mourir à domicile si tel est le souhait de la personne malade et des PA. Ainsi, il est primordial de développer une trajectoire de soins et services qui tiennent compte de ces besoins et se poursuivent durant la période de deuil. Le soutien de l'infirmière par l'enseignement sur les soins, la médication, la gestion des symptômes et le soutien émotionnel contribue à répondre aux besoins des PA en temps opportun et favorise l'établissement de la relation de confiance et potentialise le maintien et le mourir à domicile. Les études futures devraient porter sur la nécessité d'une approche intégrée précoce de soins palliatifs qui inclut les PA afin de faciliter l'accès aux soins et services appropriés en temps opportun tout au long de la trajectoire de la maladie.

Funk, L. M., Stajduhar, K. I., \& Outcalt, L. (2015). What family caregivers learn when providing care at the end of life: A qualitative secondary analysis of multiple datasets. Palliative \& Supportive Care, 13(3), 425-433. https://doi.org/10.1017/S1478951513001168

Gomes, B., \& Higginson, I. J. (2006). Factors influencing death at home in terminally ill patients with cancer: systematic review. British Medical Journal (International Edition), 332(7540), 515-521. https://doi.org/10.1136/bmj.38740.614954.55

Harding, R., Epiphaniou, E., Hamilton, D., Bridger, S., Robinson, V., George, R., ... Higginson, I. (2012). What are the perceived needs and challenges of informal caregivers in home cancer palliative care? Qualitative data to construct a feasible psycho-educational intervention. Supportive Care in Cancer, 20(9), 1975-1982. https:// doi.org/10.1007/s00520-011-1300-z

Hasson, F., Kernohan, W. G., McLaughlin, M., Waldron, M., McLaughlin, D., Chambers, H., \& Cochrane, B. (2010). An exploration into the palliative and end-of-life experiences of carers of people with Parkinson's disease. Palliative Medicine, 24(7), 731736. https://doi.org/10.1177/0269216310371414

Hébert, J., Ouellet, N., Lessard, L., Babineau, L., Veillette, A.-M., \& Coutu, M. (2017). Rapport de recherche : Conditions favorisant et limitant le maintien à domicile en soins palliatifs et de fin de vie sur le territoire du CISSS de Chaudière-Appalaches Québec : Centre intégré de santé et de services sociaux de Chaudière-Appalaches. https://www.cisss-ca.gouv.qc.ca/fileadmin/documents/Services_ offerts/Soins_palliatifs_et_de_fin_de_vie/Projet_SPFV-I_Rapport_ final_CISSS-CA_H \%C3 \%A9bert_et_al_2017-11-08.pdf 
Holm, M., Henriksson, A., Carlander, I., Wengström, Y., \& Öhlen, J. (2015). Preparing for family caregiving in specialized palliative home care: An ongoing process. Palliative a Supportive Care, 13(3), 767-775. https://doi.org/10.1017/S1478951514000558

Horseman, Z., Milton, L., \& Finucane, A. (2019). Barriers and facilitators to implementing the Carer Support Needs Assessment Tool in a community palliative care setting. British Journal of Community Nursing, 24(6), 284-290. https://doi.org/10.12968/ bjcn.2019.24.6.284

Johnson, A. (2015). Role of district and community nurses in bereavement care: A qualitative study. British Journal of Community Nursing, 20(10), 494-501. https://doi.org/10.12968/ bjcn.2015.20.10.494

Ministère de la santé et des services sociaux (2015). Soins palliatifs et de fin de vie: Plan de développement 2015-2020. http://publications. msss.gouv.qc.ca/msss/fichiers/2015/15-828-01W.pdf

L'Appui pour les proches aidants d'aînés. (2012). Portrait statistique des proches aidants de personnes de 65 ans et plus au Québec, 2012. Montréal, Québec. https://www.lappui.org/content/ download/10915/file/2016_Portrait \%20statistique.pdf

Légis Québec. (2018). Loi concernant les soins de fin de vie. Québec : Gouvernement du Québec. http://legisquebec.gouv.qc.ca/fr/ ShowDoc/cs/S-32.0001

Mason, N., \& Hodgkin, S. (2019). Preparedness for caregiving: A phenomenological study of the experiences of rural Australian family palliative carers. Health \& Social Care in the Community. https://doi.org/10.1111/hsc.12710

Meleis, A. I. (2010). Transitions theory: Middle range and situation specific theories in nursing research and practice. Springer Publishing Company.

Miles, M. B., \& Huberman, A. M. (2003). Analyse des données qualitatives. De Boeck Supérieur.

OMS/WHO. Organisation mondiale de la Santé/World Health Organization (2018). Soins palliatifs http://www.who.int/fr/ news-room/fact-sheets/detail/palliative-care

Penrod, J., Hupcey, J. E., Shipley, P. Z., Loeb, S. J., \& Baney, B. (2012). A model of caregiving through the end of life: Seeking normal. Western Journal of Nursing Research, 34(2), 174-193. https://doi. org/10.1177/0193945911400920

Robinson, C. A., Bottorff, J. L., McFee, E., Bissell, L. J., \& Fyles, G. (2017). Caring at home until death: Enabled determination. Supportive Care in Cancer, 25(4), 1229-1236. https://doi.org/10.1007/ s00520-016-3515-5
Seamark, D., Blake, S., Brearley, S. G., Milligan, C., Thomas, C. Turner, M., ... Payne, S. (2014). Dying at home: A qualitative study of family carers' views of support provided by GPs community staff. The British Journal of General Practice: The Journal of the Royal College of General Practitioners, 64(629), 796-803. https://doi org/10.3399/bjgp14X682885

Sheehy-Skeffington, B., McLean, S., Bramwell, M., O'Leary, N., \& O'Gorman, A. (2014). Caregivers experiences of managing medications for palliative care patients at the end of life: a qualitative study. The American Journal Of Hospice Q Palliative Care, 31(2), 148-154. https://doi.org/10.1177/1049909113482514

SCC/CCS. Société canadienne du cancer/Canadian Cancer Society(2013). Soins de fin de vie au Québec. Priorité aux soins palliatifs : accès, temps, lieu. (Mémoire). Société canadienne du cancer - division Québec, Québec.

Soroka, J. T., Froggatt, K., \& Morris, S. (2018). Family caregivers' confidence caring for relatives in hospice care at home: An exploratory qualitative study. The American Journal of Hospice \& Palliative Care, 35(12), 1540-1546. https://doi. org/10.1177/1049909118787779

Statistique Canada. (2019). Tableau 13-10-0715-01 Décès, selon le lieu de décès (en milieu hospitalier ou ailleurs qu'en milieu hospitalier) : Gouvernement du Canada. https://www150.statcan.gc.ca/t1/tbl1/ fr/tv.action?pid=1310071501\&pickMembers \%5B0 \%5D=1.6

Stewart, A. L., Teno, J., Patrick, D. L., \& Lynn, J. (1999). The concept of quality of life of dying persons in the context of health care. Journal of Pain and Symptom Management, 17(2), 93-108.

Totman, J., Pistrang, N., Smith, S., Hennessey, S., \& Martin, J. (2015). 'You only have one chance to get it right': A qualitative study of relatives' experiences of caring at home for a family member with terminal cancer. Palliative Medicine, 29(6), 496-507. https://doi. org/10.1177/0269216314566840

Wahid, A. S., Sayma, M., Jamshaid, S., Kerwat, D. A., Oyewole, F., Saleh, D., ... Payne, S. (2018). Barriers and facilitators influencing death at home: A meta-ethnography. Palliative Medicine, 32(2), 314-328. https://doi.org/10.1177/0269216317713427

Wilson, E., Caswell, G., Turner, N., \& Pollock, K. (2018). Managing medicines for patients dying at home: A review of family caregivers' experiences. Journal of Pain \& Symptom Management, 56(6), 962-974. https://doi.org/10.1016/j.jpainsymman.2018.08.019 